\title{
New Slip Control System Considering Actuator Dynamics
}

\author{
Amir Soltani*, Francis Assadian** \\ *Cranfield University, Cranfield, UK **University of California, Davis, USA
}

\begin{abstract}
A new control strategy for wheel slip control, considering the complete dynamics of the electro-hydraulic brake (EHB) system, is developed and experimentally validated in Cranfield University's HiL system. The control system is based on closed loop shaping Youla-parameterization method. The plant model is linearized about the nominal operating point, a Youla parameter is defined for all stabilizing feedback controller and control performance is achieved by employing closed loop shaping technique. The stability and performance of the controller are investigated in frequency and time domain, and verified by experiments using real EHB smart actuator fitted into the HiL system with driver in the loop.
\end{abstract}

\section{Introduction}

Wheel slip dynamics is characterized by highly nonlinear and uncertain behaviour of tire forces and fast changing dynamics of the wheel during braking. Because of the complex and variable dynamics of the system, design of a slip control system is a challenging task. Several linear and nonlinear control design approaches have been proposed in the literatures, ranging from linear and nonlinear PID control system [1], fuzzy logic [2], gain scheduling [3] to nonlinear methodologies such as sliding mode [4] and Lyapunov-based [5] control design approaches. However, it is worth noting that most of the proposed brake controllers, which can be found in the published literatures, consider wheel slip (and/or angular velocity) differential equations as the plant model, without taking the complete dynamics of the brake system (such as calliper and value dynamics) and their constraints into consideration. Interestingly, it is shown in [5] that it is impossible to employ a single linear PID controller for complete brake dynamics to provide stability and performance at all operational conditions.

In this paper, a new wheel slip control system based on Youla parameterization approach is proposed [6]. The controller provides stability and good control performance over the whole range of operating conditions of the system, considers all the existing dynamics and constraints of the brake system. In the next sections, the mathematical model of the system is introduced, then the proposed closed loop control system based on Youla parameterization is presented, and finally the stability and performance of the control system is validated by simulation and HiL testing at different driving conditions.

\section{System Modelling}

A new control law for continues control of wheel slip is presented in this paper. It is assumed that the smart brake actuation (i.e. brake pressure control) is already provided by an off-the-shelf electro hydraulic brake (EHB) system, also called Sensotronic Brake Control (SBC), which has been developed by Daimler and Bosch [7]. Schematic diagram of Bosch EHB SBC system is shown in Fig 1 [8].

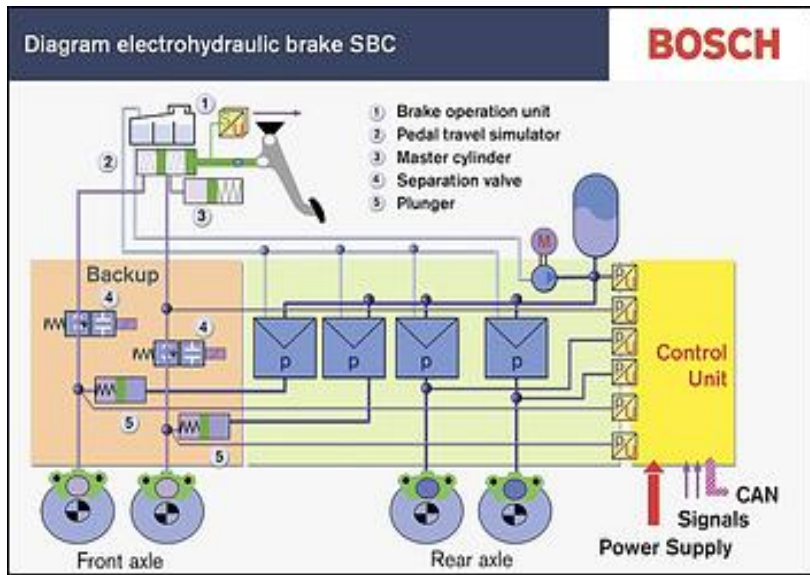

Figure 1: Schematic diagram of Bosch SBC system [8]

Employing electro hydraulic brake system as a smart brake actuator provides the possibility to control individual wheel brake line pressure to follow the desired target pressure continuously [9]. The relevant reference brake pressure for each wheel (to be generated by EHB system) is provided by the proposed slip control system. Having continuous control over brake pressure provides several advantages such as driver comfort as well as the possibility of fast and precise control over the tires longitudinal force (and slip) [10].

As the brake pressure is regulated for each wheel individually (through EHB valve modulation unit, as shown in Figure 1), there are 4 independent inputs and 4 similar plants, and four similar SISO closed loop brake control systems exist in this architecture. Design of the closed loop brake control system based on wheel slip feedback for one wheel ( $i-t h$ wheel), as shown in Figure 2, is presented in this section. 


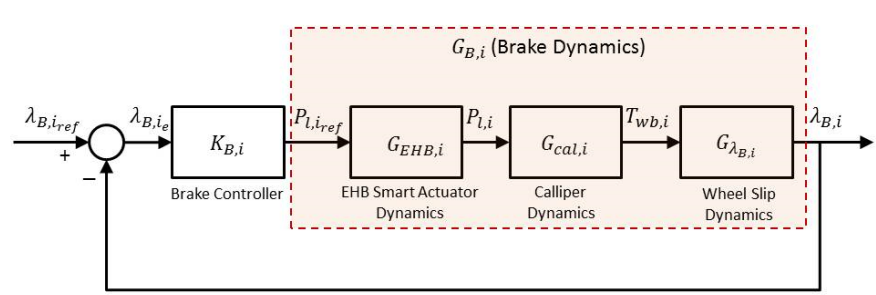

Figure 2: Closed Loop Brake Control system

The starting point in designing the control system is to derive the plant dynamics model. Here the plant, so called single corner wheel model, consists of the quarter of car mass moving in $x$ direction with a single independent rotating wheel subject to braking torque $T_{b, i}$ at the wheel hub (centre) and longitudinal force $F_{x, i}$ at the centre of tireground contact patch, as shown in Figure 3. It should be noted that the quarter car model relies on some simplifications; however, this is a simple and effective model which considers the major braking dynamics and is widely applied in active braking control system designs $[4,5,11]$.

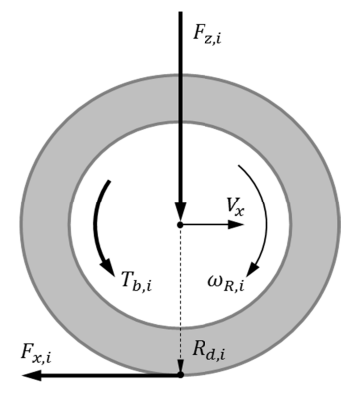

Figure 3: Wheel Dynamics Model

The equation of wheel dynamics is:

$$
J_{y, i} \dot{\omega}_{R, i}=T_{b, i}-R_{d, i} F_{x, i}
$$

where $J_{y, i}$ is the $(i-t h)$ wheel moment of inertia, $R_{d, i}$ is the wheel radius and $\omega_{R, i}$ is the wheel angular velocity. The quarter car dynamics in the longitudinal direction can be written as:

$$
-\dot{V}_{x} m_{s}=-F_{x, i}
$$

where $m_{s}$ is the quarter car mass (as described above) and $\dot{V}_{x}$ is the vehicle longitudinal acceleration. The tire longitudinal force $F_{x, i}$ is a nonlinear function of $\operatorname{slip} \lambda_{B, i}$, i.e

$$
F_{x, i}\left(\lambda_{B, i}\right)=\mu_{x, i}\left(\lambda_{B, i}\right) F_{z, i}
$$

The tire slip ratio during braking $\lambda_{B, i}$ was defined as [7]:

$$
\lambda_{B, i}=\frac{R_{d, i} \omega_{R, i}}{V_{x, i}}-1 \quad\left(-1 \leq \lambda_{B, i} \leq 0\right)
$$

where $V_{x, i}$ is the forward speed of the tire centre which is here assumed that is equal to vehicle longitudinal speed $V_{x}$.

Combining Eq. (1) with Eq. (4), the relationship between braking torque input and tire slip output can be derived as:

$$
\dot{\lambda}_{B, i}=-\frac{1}{V_{x}}\left(\dot{V}_{x}\left(\lambda_{B, i}+1\right)+\frac{R_{d, i}^{2}}{J_{y, i}} F_{x, i}\right)+\frac{R_{d, i}}{J_{y, i} V_{x}} T_{w b, i}
$$

Substituting $\dot{V}_{x}$ from Eq. (2) into Eq. (5), the dynamics of the system can be formulated as a first order model of the wheel slip dynamics as:

$$
\dot{\lambda}_{B, i}=-\frac{1}{V_{x}}\left(\frac{\left(\lambda_{B, i}+1\right)}{m_{s}}+\frac{R_{d, i}^{2}}{J_{y, i}}\right) F_{x, i}+\frac{R_{d, i}}{J_{y, i} V_{x}} T_{w b, i}
$$

Eq. (6) is a nonlinear function of two state variables $V_{x}$ and $\lambda_{B, i}$.

To be able to design a linear control system, one should linearize Eq. (6) around nominal operating points $V_{x, n}$ and $\lambda_{B, i, n}$ (we will discuss this nominal operating point in the following paragraphs).

Considering $\Delta \lambda_{B, i}=\lambda_{B, i}-\lambda_{B, i, n}$ and $\Delta V_{x}=V_{x}-V_{x, n}$ and $\Delta T_{w b, i}=$ $T_{w b, i}-T_{w b, i, n}$; the linear transfer function for plant dynamics (from brake torque input to tire slip output) can be derived by application of first order Tylor series expansion as [5]

$$
\begin{aligned}
& G_{\lambda_{B, i}}(s)=\frac{\lambda_{B, i, n}}{T_{w b, i, n}} \\
& =\frac{R_{d, i}}{J_{y, i} V_{x, n}}\left[\frac{1}{s+\frac{\dot{\mu}_{x, i, n} F_{z, i}}{m_{s} V_{x, n}}\left(\left(1+\lambda_{B, i, n}\right)+\frac{m_{s} R_{d, i}^{2}}{J_{y, i}}\right)}\right]
\end{aligned}
$$

where $\dot{\mu}_{x, i, n}\left(\lambda_{B, i, n}\right)=\left.\frac{\partial \mu_{x, i}}{\partial \lambda_{B, i}}\right|_{\lambda_{B, i, n}}$, represents the slope of the $\mu_{x, i}$ curve around a nominal operating point. It should be noted that calculation of $\dot{\mu}_{x, i, n}$ in Eq.(7) is not dependent on any specific tire model. It could be obtained either by differentiation of a tire model, such as Burckhardt tire model as cited in the literatures (see [12] for example), or by implementation of an online algorithm to detect the sign of the friction curve slope, which is suitable for practical applications [5,13].

The linearized plant (7) (herein after called plant) is of first order in form of:

$$
G_{\lambda_{B, i}}(s)=\frac{k_{i}}{s+p_{i}}
$$

with a gain of:

$$
k_{i}=\frac{R_{d, i}}{J_{y, i} V_{x, n}}
$$

and a single pole of: 


$$
p_{i}=\frac{\dot{\mu}_{x, i, n} F_{z, i}}{m_{s} V_{x, n}}\left(\left(1+\lambda_{B, i, n}\right)+\frac{m_{s} R_{d, i}^{2}}{J_{y, i}}\right)
$$

To derive a control oriented linear plant model, it is essential to select an appropriate operating point, where the plant is being linearized around it. By studying the slip dynamics transfer function, Eq. (7), one can conclude that the tire normal load, $F_{z, i}$, the vehicle velocity, $V_{x, n}$, and the road condition (which is reflected on $\dot{\mu}_{x, i, n}$ ) are the main parameters that are considerably changing during braking actuation time period ${ }^{1}$. Therefore, to investigate the variation of plant dynamics, it is required to derive the maximum and minimum values of longitudinal speed $V_{x}, F_{z, i}$ and $\dot{\mu}_{x, i}$ in the plant dynamics transfer function, Eq. (7).

As $V_{x}$ is always positive (forward driving assumption), its variation cannot make the plant unstable, but, it will impact the value of gain $\left(k_{i}\right)$. On the other hand, the change of $F_{z, i}$ and $\dot{\mu}_{x, i, n}$ will move the location of pole on $s$ plan, which will have an effect on the stability of plant. More specifically, the effect of $\dot{\mu}_{x, i, n}$ variation on the plant dynamics is significant as it can make the plant unstable, as discussed below.

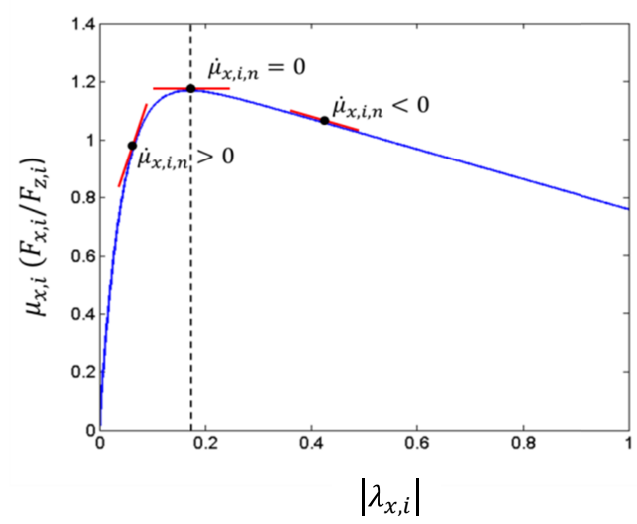

Figure 4: Slope of $\mu(\mathrm{x}, \mathrm{i})$ at different operating points

Considering a typical longitudinal tire force/slip curve, as shown in Figure 4, one can conclude from Eq. (10) that the open loop transfer function $G_{\lambda_{B, i}}(\mathrm{~s})$ is stable before slip peak (as $\dot{\mu}_{x, i, n}>0$, therefore $\left.p_{i}>0\right)$, and become unstable when the slip goes beyond the peak point (as $\dot{\mu}_{x, i, n}<0$, therefore $p_{i}<0$ ). Therefore, the worst case operating points are corresponding to high longitudinal speed $V_{x}$, low normal load $F_{z, i}$ and smallest positive slope of $\mu_{x, i}$ which is equivalent to the smallest distance of stable pole from imaginary axis in $s$ plan. Moreover, when the linearization point is close to the peak of the curve, i.e. $\dot{\mu}_{x, i, n} \cong 0$, the plant becomes a pure integrator:

$$
G_{\lambda_{B, i}}(s)=\frac{k_{i}}{s}
$$

\footnotetext{
${ }^{1}$ By other word, it is assumed that the vehicle mass, wheel inertia, and wheel radius are constant.
}

To obtain the value of tire friction slope, $\dot{\mu}_{x, i}\left(\lambda_{B}\right)$, one can employ the Burckhardt tire model [12]. Based on Burckhardt tire model, the relationship between tire slip and normalised longitudinal tire force is defined as:

$$
\mu_{x, i}\left(\lambda_{B, i}\right)=\frac{F_{x, i}\left(\lambda_{B, i}\right)}{F_{z, i}}=c_{1}\left(1-e^{-c_{2} \lambda_{B, i}}\right)-c_{3} \lambda_{B, i}
$$

where $c_{1}, c_{2}$ and $c_{3}$ are constants, as defined for different road surfaces in Table 1 . Therefore

$$
\dot{\mu}_{x, i}\left(\lambda_{B, i}\right)=\frac{\partial \mu_{x, i}\left(\lambda_{B, i}\right)}{\partial \lambda_{B, i}}=c_{1} c_{2} e^{-c_{2} \lambda_{B, i}}-c_{3}
$$

Table 1: Values of the Burckhardt tire model coefficients [12]

\begin{tabular}{|l|c|c|c|}
\hline Road Surface & $\boldsymbol{c}_{\mathbf{1}}$ & $\boldsymbol{c}_{\mathbf{2}}$ & $\boldsymbol{c}_{\mathbf{3}}$ \\
\hline Asphalt, dry & 1.2801 & 23.99 & 0.52 \\
\hline Asphalt, wet & 0.857 & 33.822 & 0.347 \\
\hline Concrete, dry & 1.1973 & 25.168 & 0.5373 \\
\hline Cobblestone, dry & 1.3713 & 6.4565 & 0.6691 \\
\hline Snow & 0.1946 & 94.129 & 0.0646 \\
\hline Ice & 0.05 & 306.39 & 0 \\
\hline
\end{tabular}

By investigating different road surfaces at different slips, one can conclude that the maximum and minimum values of $\dot{\mu}_{x, i}$ happen on dry asphalt at $\lambda_{B, i}=0$ with the value of 30.2 and on dry cobblestone at slip $\left|\lambda_{B, i}\right|=1$ with the value of -0.658 , respectively. Therefore the slip slope bound is:

$$
-0.658 \leq \dot{\mu}_{x, i} \leq 30.2
$$

The tire normal load on each wheel $F_{z, i}$ is a function of the vehicle weight distribution (static load) and load transfer due to longitudinal and lateral accelerations (dynamic loads), which can be estimated from the following equations [14]:

$$
\begin{aligned}
& F_{z, 1}=\left[\frac{1}{2} m g \frac{l_{r}}{\left(l_{f}+l_{r}\right)}\right]-\left[\frac{1}{2} m a_{x} \frac{h}{\left(l_{f}+l_{r}\right)}\right]-\left[\frac{1}{2} m a_{y} \frac{h}{l_{w}}\right] \\
& F_{z, 2}=\left[\frac{1}{2} m g \frac{l_{r}}{\left(l_{f}+l_{r}\right)}\right]-\left[\frac{1}{2} m a_{x} \frac{h}{\left(l_{f}+l_{r}\right)}\right]+\left[\frac{1}{2} m a_{y} \frac{h}{l_{w}}\right] \\
& F_{z, 3}=\left[\frac{1}{2} m g \frac{l_{r}}{\left(l_{f}+l_{r}\right)}\right]+\left[\frac{1}{2} m a_{x} \frac{h}{\left(l_{f}+l_{r}\right)}\right]-\left[\frac{1}{2} m a_{y} \frac{h}{l_{w}}\right] \\
& F_{z, 4}=\left[\frac{1}{2} m g \frac{l_{r}}{\left(l_{f}+l_{r}\right)}\right]+\left[\frac{1}{2} m a_{x} \frac{h}{\left(l_{f}+l_{r}\right)}\right]+\left[\frac{1}{2} m a_{y} \frac{h}{l_{w}}\right]
\end{aligned}
$$

where $m$ is the total sprung and unsprung vehicle mass, $l_{f}$ and $l_{r}$ are front and rear tire distance to Centre of Gravity (CG), respectively, $l_{w}$ is the track width, $h$ is the height of CG from the ground and indices $(i=1,2,3,4)$ refer to front left, front right, front left, and rear left wheels, respectively. 


\begin{tabular}{|l|c|l|c|}
\hline Parameters & Abbreviation & value & Unit \\
\hline Vehicle Mass & $m$ & 1226 & $\mathrm{~kg}$ \\
\hline Front Tire distance to Centre of Gravity & $l_{f}$ & 0.863 & $\mathrm{~m}$ \\
\hline Rear Tire distance to Centre of Gravity & $l_{r}$ & 1.567 & $\mathrm{~m}$ \\
\hline Half track & $l_{w}$ & 0.71 & $\mathrm{~m}$ \\
\hline Height of CG from the ground & $h$ & 0.519 & $\mathrm{~m}$ \\
\hline Vehicle Inertia & $I_{z}$ & 1458.76 & $\mathrm{~kg} \mathrm{~m}$ \\
\hline Gravitational Acceleration & $g$ & 9.8 & $\mathrm{~m} / \mathrm{s}^{2}$ \\
\hline Wheel inertia & $I_{y, i}$ & 1.17 & $\mathrm{~kg} \mathrm{~m}$ \\
\hline Wheel dynamic radius & $R_{d y n}$ & 0.266 & $\mathrm{~m}$ \\
\hline Brake gain factor (front) & $K_{b, i}, i=1,2$ & 10 & $\mathrm{Nm} / \mathrm{bar}$ \\
\hline Brake gain factor (rear) & $K_{b, i}, i=3,4$ & 5 & $\mathrm{Nm} / \mathrm{bar}$ \\
\hline line pressure build up time lag & $\tau_{c a l}$ & 0.1 & $\mathrm{sec}$ \\
\hline EHB actuator build up time lag & $\tau_{E H B}$ & 0.1 & $\mathrm{sec}$ \\
\hline
\end{tabular}

Considering the vehicle parameters, as indicated in Table 2, and assuming the extreme magnitudes of $a_{x}=-g$ and $a_{y}=g$, the maximum normal force will be applied on the front right tire is:

$$
F_{z_{\max }}=F_{z, 2}=0.823 \mathrm{mg}=9898.3 \mathrm{~N} \approx 10 \mathrm{KN}
$$

The same conclusion can be made when $a_{y}=-g$, however, the maximum normal force will be applied to front left tire $\left(F_{z, 1}\right)$, instead. Therefore the normal force range limit is:

$$
0 \leq F_{z, i} \leq 10 K N
$$

Finally, it is assumed that the longitudinal velocity range (in which the safety brake actuation will be activated) is between 10 to $50 \mathrm{~m} / \mathrm{s}$, i.e.

$$
10 \leq V_{x} \leq 50 \mathrm{~m} / \mathrm{s}
$$

Employing the above operational limits of $\dot{\mu}_{x, i}, F_{z, i}$ and $V_{x}$, the maximum and minimum values of gain and pole of the plant (for the vehicle with the values indicated in Table 2) can be obtained from Eqs. (9) and (10), respectively. By investigating the possible magnitudes of the plant's gain and pole locations, it is concluded that the plant dynamics is highly sensitive to its operating conditions. Therefore, selection of appropriate nominal operating point plays an important role in design of the proposed control system.

Finally, it should be noted that the complete brake plant model (i.e. from pressure input to wheel slip output, as shown in Figure 1), includes the slip dynamics, the calliper dynamics and the EHP smart actuator dynamics. The required barking torque about the wheel spin axis and the subsequence braking force is produced by application of hydraulic brake pressure at the brake callipers. The relationship between brake line pressure $P_{l, i}$ and wheel (bake) torque $T_{w b, i}$ is defined as [11]:

$$
T_{w b, i}=K_{b, i} P_{l, i}\left(\frac{1}{\tau_{c a l} s+1}\right)
$$

where $K_{b, i}$ and $\tau_{c a l}$ are the calliper gain and time constant (time lag), respectively. The calliper dynamics $G_{C a l, i}(\mathrm{~s})$ is

$$
G_{c a l, i}=\frac{T_{w b, i}}{P_{l, i}}=\frac{K_{b, i}}{\tau_{c a l} S+1}
$$

Similarly, the EHB smart actuator dynamics $G_{E H B, i}(\mathrm{~s})$ can be considered as a stable first order transfer functions as [21]:

$$
G_{E H B, i}(s)=\frac{1}{\tau_{E H B} s+1}
$$

where $\tau_{E H B}$ is the EHB time constant (time lag). Therefore, the complete plant dynamics takes the form of:

$$
\begin{gathered}
G_{B, i}(s)=G_{\lambda_{B, i}}(s) G_{E H B, i}(s) G_{C a l, i}(s) \\
G_{B, i}(s)=\left(\frac{k_{i}}{s+p_{i}}\right)\left(\frac{K_{b, i}}{\tau_{c a l} s+1}\right)\left(\frac{1}{\tau_{E H B} s+1}\right) \\
=\frac{k_{i} \times K_{b, i}}{\left(s+p_{i}\right)\left(\tau_{c a l} s+1\right)\left(\tau_{E H B} s+1\right)}
\end{gathered}
$$

Bode diagram of the plant dynamics at different longitudinal slips is plotted in Figure 5, assuming the vehicle and brake parameters as indicated in Table 2, $\tau_{c a l}=\tau_{E H B}=0.1 \mathrm{sec}$ [11], dry asphalt, $F_{z, i}=10 \mathrm{KN}$ and $V_{x}=50 \mathrm{~m} / \mathrm{s}$. By investigating the phase angle of the plant, it is clear that the plant is stable at the slip values lower than $\lambda_{x, i}=0.16$ and become unstable at higher slips. As discussed before, the tire slip of $\lambda_{x, i}=0.16$ corresponds to the peak point of the tire friction curve on dry asphalt, where $\dot{\mu}_{x, i}=0$ (see also Figures 4).

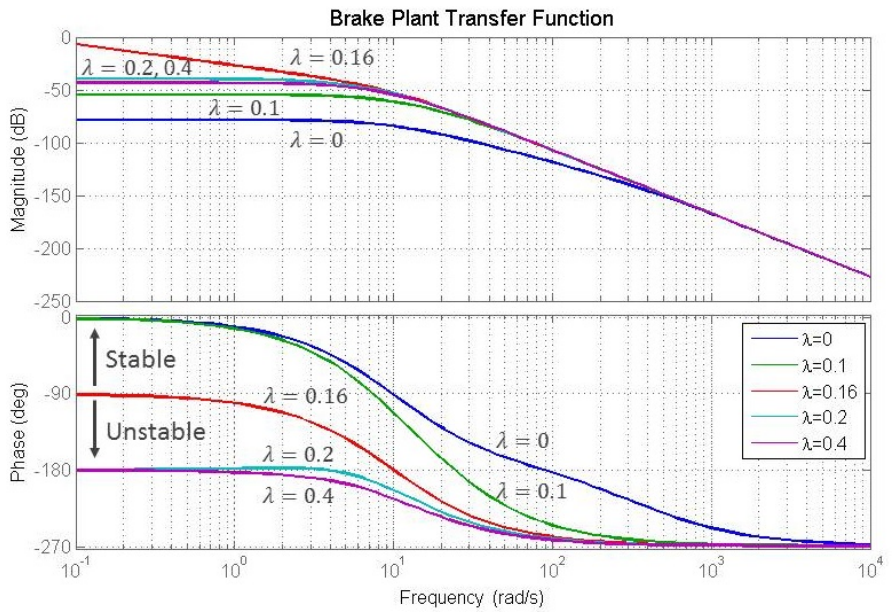

Figure 5: Plant dynamics $\mathrm{G}_{\mathrm{B}, \mathrm{i}}$ for different slips (dry asphalt, $\mathrm{F}_{\mathrm{z}, \mathrm{i}}=10 \mathrm{KN}$ and $\left.\mathrm{V}_{\mathrm{x}}=50 \mathrm{~m} / \mathrm{s}\right)$

\section{Control System Design}

Due to the fact that the dynamics of the system is changing considerably during its operational envelope, and there are several uncertainties that exist in the system (such as brake pad coefficient of friction and so on); it is necessary to employ a feedback control system, as shown in Figure 2, to provide stability as well as good performance for the system at all operating conditions. In this paper, 
a new closed loop wheel slip control system based on Youla parameterization approach is proposed [15, 16, and 17].

Investigating the complete (linearized) plant dynamics, as described by Eq. (21), one can conclude that the plant dynamics consists of three first order transfer functions. We take the Youla parameter as the inverse of the plant transfer function at a nominal operating point, $G_{B, i, n}$, multiply to three stable first order filters with adjustable poles corresponding to the three dynamics exist in the system, such as:

$$
Y_{B, i}=\frac{1}{G_{B, i, n}}\left[\frac{1}{\left(\tau_{1} s+1\right)\left(\tau_{2} s+1\right)\left(\tau_{3} s+1\right)}\right], \quad \tau_{1}, \tau_{2}, \tau_{3}>0
$$

By selecting a stable nominal plant $G_{B, i, n}$, the proposed Youla transfer function is stable (and minimum phase), therefore, internal stability of the feedback system is guaranteed [17]. The tuneable parameters $\tau_{1}, \tau_{2}$ and $\tau_{3}$ can be employed to shape of the closed loop transfer functions $S_{B, i}$ and $T_{B, i}$ and control system bandwidth such that it could provide robust performance considering plant dynamics uncertainties at low frequencies and attenuate sensor noise at high frequencies.

The complementary sensitivity $T_{B, i}$ and sensitivity $S_{B, i}$ transfer functions are:

$$
\begin{gathered}
T_{B, i}=Y_{B, i} \times G_{B, i, n}=\frac{1}{\left(\tau_{1} s+1\right)\left(\tau_{2} s+1\right)\left(\tau_{3} s+1\right)} \\
S_{B, i}=1-T_{B, i, n}=\frac{\left(\tau_{1} s+1\right)\left(\tau_{2} s+1\right)\left(\tau_{3} s+1\right)-1}{\left(\tau_{1} s+1\right)\left(\tau_{2} s+1\right)\left(\tau_{3} s+1\right)}
\end{gathered}
$$

The controller transfer function $K_{B, i}$ can be derived as:

$$
K_{B, i}=\frac{Y_{B, i}}{S_{B, i}}=\frac{1}{G_{B, i, n}\left[\left(\tau_{1} s+1\right)\left(\tau_{2} s+1\right)\left(\tau_{3} s+1\right)-1\right]}
$$

and the open loop transfer function $L_{B, i}$ is:

$$
L_{B, i}=K_{B, i} \times G_{B, i, n}=\frac{1}{\left[\left(\tau_{1} s+1\right)\left(\tau_{2} s+1\right)\left(\tau_{3} s+1\right)-1\right]}
$$

Recall, $G_{B, i, n}$, is the linearized transfer function of plant dynamics at a nominal operating point where the nonlinear differential equation of the slip dynamics was linearized around its nominal point. As explained before, the dynamics and stability of the plant is highly dependent on these parameters; therefore, selecting different operating points results in Youla parameters (and controllers) with different behaviours. To obtain an appropriate plant dynamics, $G_{B, i, n}$, (for our proposed control design approach), the nominal operating point for $\dot{\mu}_{x, i, n}$ should be selected at a slip value, $\lambda_{x, i, n}$, where the tire friction curve $\mu_{x, i}$ is near to its peak value but is in stable region (i.e $\dot{\mu}_{x, i}>0$ ). Note that the wheel slip value corresponding to the abscissa of the maximum of $\mu_{x, i}$ is different at various road surfaces, as illustrated in Figure 6.

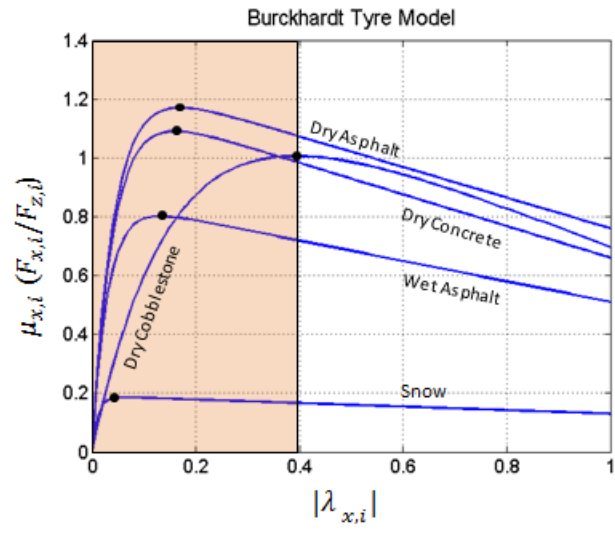

Figure 6: Longitudinal friction curve

Taking the nominal operating points as: dry asphalt friction curve (from Burckhardt tire model), $\lambda_{x, i, n}=0.09, F_{z, i, n}=1 / 4 \mathrm{mg} N$ and $V_{x, n}=10 \mathrm{~m} / \mathrm{s}, \tau_{E H B}=\tau_{c a l}=0.1 \mathrm{sec}$ and $\tau_{1}=\tau_{2}=\tau_{3}=\tau_{B}$; the control system transfer functions (for the vehicle parameters as indicated in Table 2) can be derived as:

$$
\begin{gathered}
G_{B, i}=\frac{5.6838}{(s+177)(s+10)^{2}} \\
T_{B, i}=\frac{1}{\left(\tau_{B} s+1\right)^{3}} \\
S_{B, i}=\frac{s\left(\tau_{B}^{3} s^{2}+3 \tau_{B}^{2} s+3 \tau_{3}\right)}{\left(\tau_{B} s+1\right)^{3}}
\end{gathered}
$$

$$
\begin{aligned}
Y_{B, i} & =\frac{0.1759(s+177)(s+10)^{2}}{\left(\tau_{B} s+1\right)^{3}} \\
K_{B, i} & =\frac{0.1759(s+177)(s+10)^{2}}{s\left(\tau_{B}^{3} s^{2}+3 \tau_{B}^{2} s+3 \tau_{B}\right)}
\end{aligned}
$$

$$
L_{B, i}=\frac{1}{s\left(\tau_{B}^{3} s^{2}+3 \tau_{B}^{2} s+3 \tau_{B}\right)}
$$

To shape the close loop response of the system, tuning parameter $\tau_{B}$ can be employed. The magnitude Bode plot of $T_{B, i}$ and $S_{B, i}$ transfer functions for two different values of $\tau_{B}$ time constant are shown in Figure 7 . The system bandwidth increase by decreasing the time constant, however, the peak values of $S$ and $T$ transfer functions $\left(M_{S}\right.$ and $M_{T}$ ) are less than $2 \mathrm{db}$ so the $6 \mathrm{db}$ gain margin is guaranteed which means that good control performance is met [19]. Moreover, the crossover gain is less than zero which means the minimum of $60^{\circ}$ phase margin is also guaranteed [20]. 


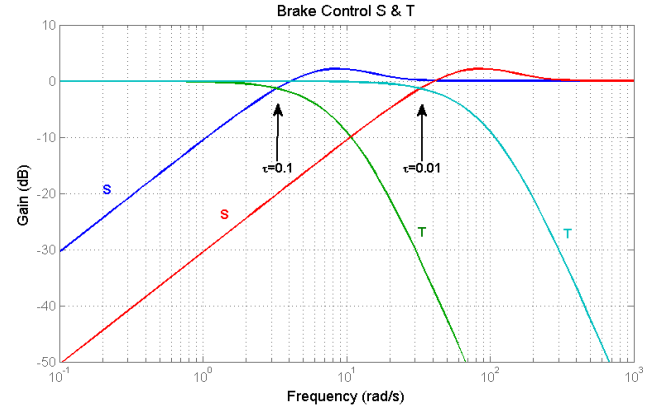

Figure 7: Brake Control System S \& T

By selecting $\tau_{B}=0.0085$, the brake controller takes the form of:

$$
K_{B, i}=\frac{2.1487 e 5(s+9.41)(s+10)^{2}}{s\left(s^{2}+352.9 s+4.152 e 4\right)}
$$

All the transfer functions of the brake control system, including plant, Youla parameter, controller, sensitivity, open loop and closed loop transfer functions, are shown in Figure 8, confirm our previous conclusion for the control system performance.

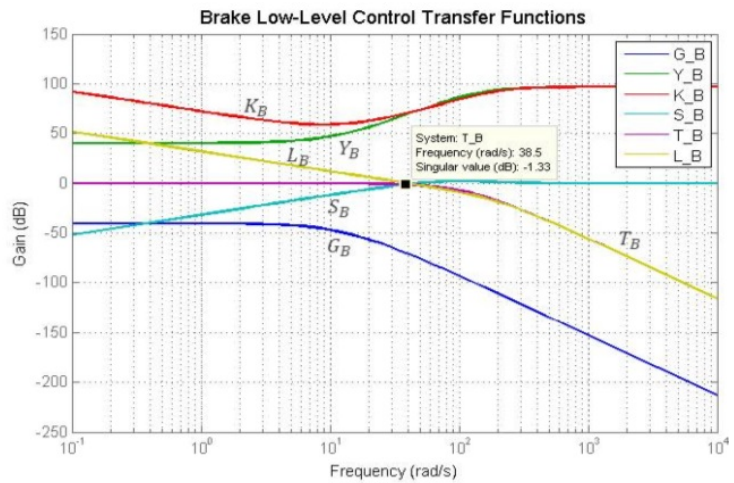

Figure 8: Frequency response of the brake control transfer functions

To investigate the behaviour of the control system in time domain, the response of the closed loop control system subject to unit step input at nominal operating point is shown in Figure 9. The result confirms a good dynamic response of the controlled linear system with no overshoot.

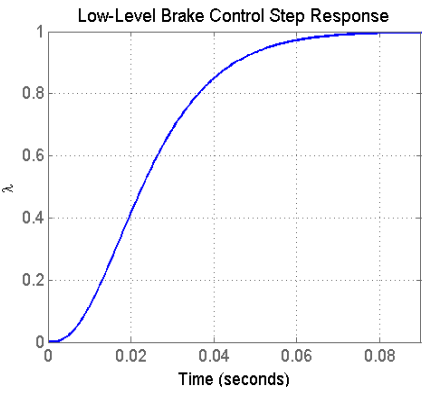

Figure 9: Unit step response of the brake control at nominal operating point

Page 6 of 8
It is worth mentioning that the proposed controller was designed based on the linearized plant transfer function at a nominal operating condition (dry asphalt friction curve (Burckhardt tire model, $\lambda_{x, i, n}=$ $0.09, F_{z, i, n}=1 / 4 m g N$ and $V_{x, n}=10 \mathrm{~m} / \mathrm{s}$ ). However, the dynamics of the plant is highly sensitive to variation of the parameters such as road surfaces, tire slip, vehicle velocity and tire normal forces, as discussed before. More importantly, increasing the tire slip (above the peak point of tire friction cure) make the plant unstable. To investigate the stability and robustness of the control system at the entire range of operational envelope, the response of closed loop brake control system subject to slip step input at different operational conditions and surfaces are plotted in Figure 10. Interestingly, the controller can stabilize the closed loop system on different surfaces and at all conditions, even in the worse-case conditions in which the plant is unstable (i.e. at tire high slip and high normal load, and vehicle low longitudinal velocity). Meanwhile, the performance of the control system exhibits a sizable variation (from underdamped to underdamped (oscillatory) behaviour). Moreover, the settling time of the system range from few millisecond to one second, depending also on the vehicle speed and normal load. This is due to utilizing a fixed structure controller for the whole ranges of the system operations, at which the dynamics of the plant is changing considerably. However, considering the fact that the controller can stabilise the plant at all conditions and track the reference value within few milliseconds in most cases (except in worse case scenarios, which cannot happen in reality), the utilisation of one fixed structure controller could be justified.
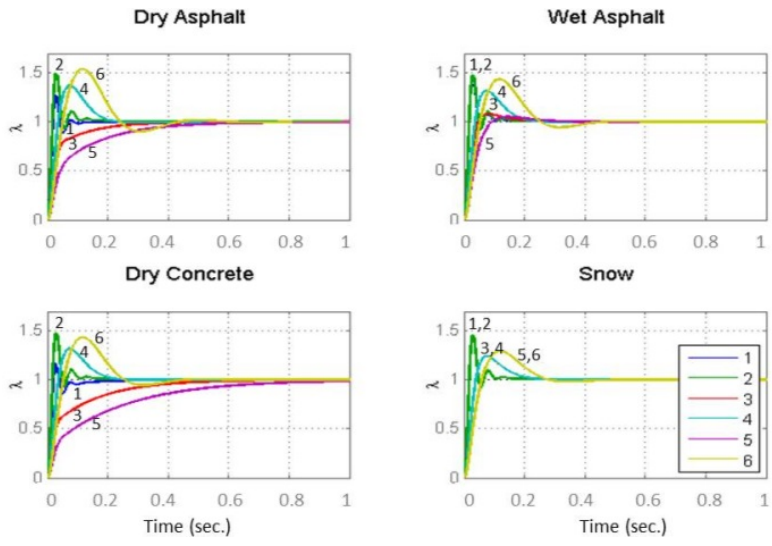

$\begin{array}{cccc} & V_{x}(m / s) & F_{z, i}(K N) & \lambda_{x, i} \\ 1 & 10 & 1 & 0.1 \\ 2 & 10 & 1 & 0.5 \\ 3 & 30 & 5 & 0.1 \\ 4 & 30 & 5 & 0.5 \\ 5 & 50 & 10 & 0.1 \\ 6 & 50 & 10 & 0.5\end{array}$

Figure 10: Brake control step response at various operational conditions

\section{Control System Validation}

\section{Simulation Results}

To examine the stability and performances of the proposed closed loop slip control system in simulation, a single wheel model, as described by Eqs. (1) and (2), in conjunction with the EHB and calliper dynamics is constructed in Simulink $₫$ environment. The brake actuator constraint is also included in the model, by limiting the pressure command to EHB within the range of $[0,200]$ bar, as shown in Figure 11. 


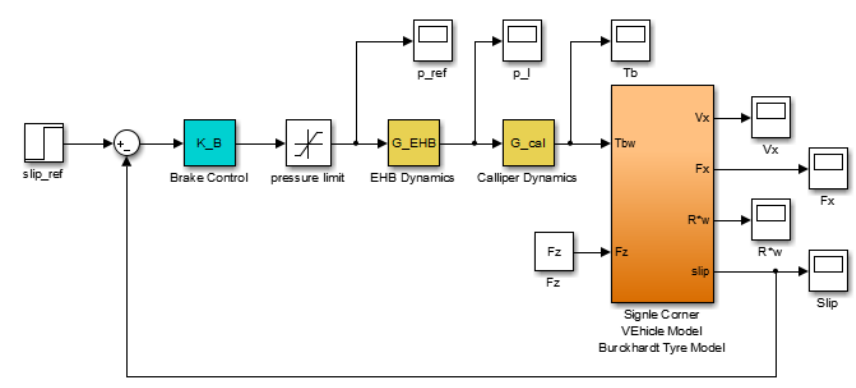

Figure 11: Closed loop brake control with brake dynamics and constraint, and quarter car vehicle dynamics

The results of simulations subject to two different driving conditions on dry asphalt surfaces are presented in Figure 12. The first driving condition $\left(V_{x}=10 \mathrm{~m} / \mathrm{s}, F_{z, i}=m g / 4 \mathrm{~N}\right.$, and $\left.\lambda_{x, i}=0.05\right)$ corresponds to low vehicle speed and nominal tire load where the slip is below its threshold limit, therefore the plant is stable. In this scenario, the slip reach to its target $\left(\lambda_{x, i}=0.05\right)$ within $1 \mathrm{sec}$ with an overdamped response and the vehicle stops within $2.3 \mathrm{sec}$. The commanded brake pressure and the tire longitudinal force are within their limits. The second driving condition $\left(V_{x}=50 \mathrm{~m} / \mathrm{s}, F_{z, i}=\right.$ $m g / 2 N$, and $\lambda_{x, i}=0.25$ ) corresponds to a severe driving condition where the tire is operating beyond its saturation limit, therefore the plant is highly unstable. It could be observed that the control system can stabilise the plant even at slips greater than 0.16 , however, due to actuator saturation, the system exhibits an overshoot, but it finally could track the reference value within $2 \mathrm{sec}$. The slip overshoots, which is clearly reflected from the difference between vehicle speed and tire longitudinal speed $\left(R_{d, i} \times \omega_{R, i}\right)$, is generated because of the brake pressure has reached its limit of 200 bar. In spite of the fact that the tire slip is in unstable region and also brake pressure is saturated; the control system is stable and can reduce the vehicle speed from 50 $\mathrm{m} / \mathrm{s}$ to 0 in less than $4 \mathrm{sec}$ (without locking the wheel).
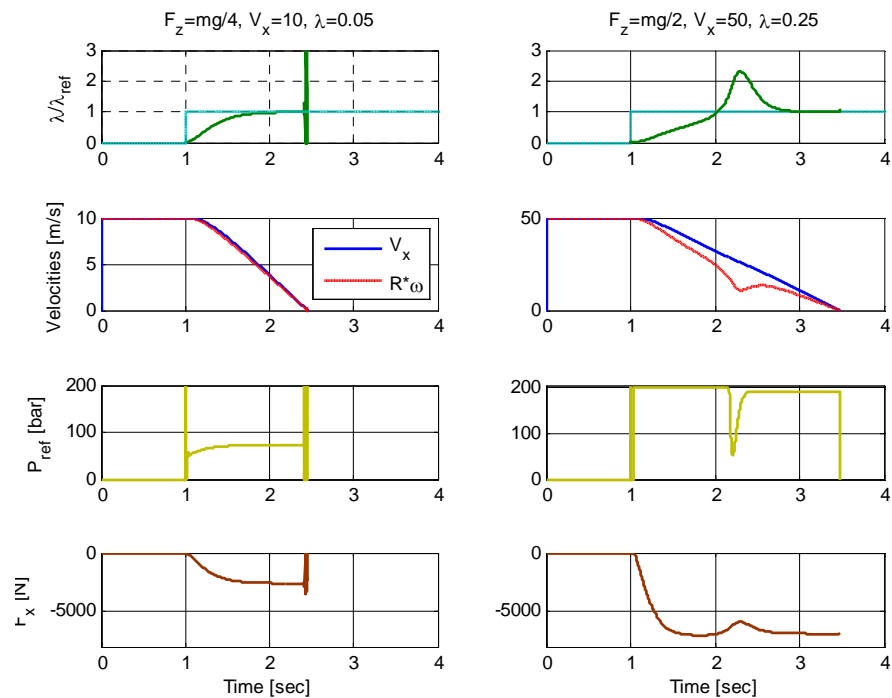

Figure 12: Brake control step response, dry asphalt

To investigate the performance of the control system on low mu surfaces, a similar simulation is performed with the same operational conditions, but on snow. The simulation results, as shown in Figure 13 , confirm the stability and performance of the control system at low speeds. Moreover, the control system can stabilize the plant and provide acceptable tracking (but with high overshoot) even in severe driving conditions.
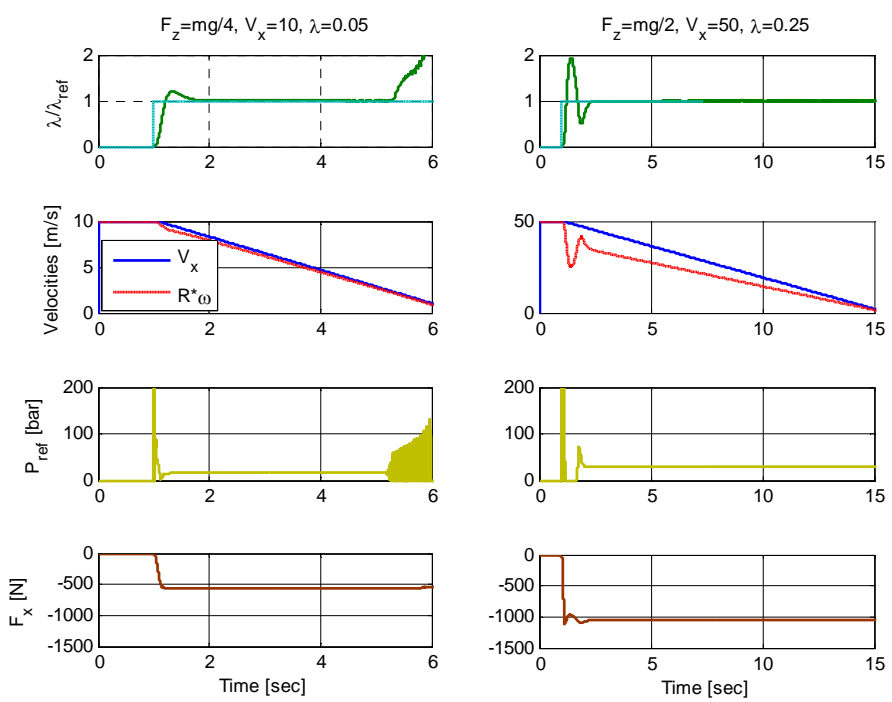

Figure 13: Brake control step response, snow

\section{HiL Testing Results}

To validate the proposed control system in real time environment with the existence of real dynamics of the brake system, the control system is implemented in dSPACE MicroAutoBox rapid control prototyping (RCP) platform in the Cranfield's integrated Brake \& Steering HiL rig system [21]. The HiL rig consists of a complete real brake system (including brake pedal, master cylinder, disk, calliper and the EHB unit), integrated with IPG CarMaker/HiL® as an offthe-shelf high fidelity real time vehicle model [22] running in a dSPACE ds1006 Simulator and a driver in the loop facility to form a comprehensive vehicle dynamic rapid control development platform.

The vehicle is driving at speed of around $33 \mathrm{~m} / \mathrm{s}(120 \mathrm{Kph})$ was subjected to step slip input (slip target was set to 0.03). The test results, as shown in Figure 14, confirm the good stability and performance of the slip control system. The brake pressure increase to 40 bar and the wheel slip reach to its target within $0.5 \mathrm{sec}$, and as a result, the vehicle longitudinal speed reduces (from $33 \mathrm{~m} / \mathrm{s}$ ) to $10 \mathrm{~m} / \mathrm{s}$ within $1 \mathrm{sec}$ without wheel locking. 

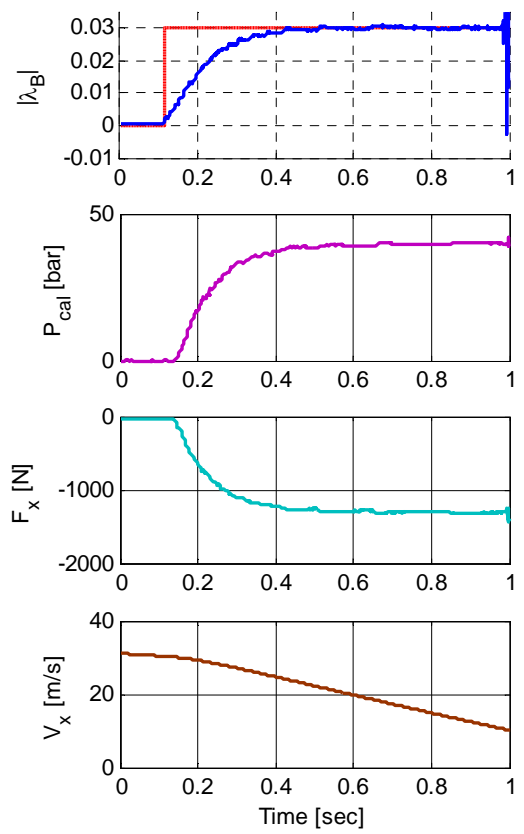

Figure 14: Closed loop slip control responses

\section{Summary/Conclusions}

A new control strategy for wheel slip control, considering the complete dynamics and constraints of the electro-hydraulic brake (EHB) system, is proposed. The control system is based on closed loop shaping Youla-parameterization method. The stability and performance of the controller are experimentally validated utilizing Cranfield University's HiL system. The real time experiments include the proposed control system implemented in a MicroAutoBox rapid control prototyping platform, with the existence of real EHB smart actuator integrated with CarMaker/HiL ${ }^{\circledR}$ vehicle model and driver in the loop (driving simulator) facility. The experimental results, verified the stability and good performances of the slip control system, even in hazardous driving conditions. The proposed slip control system can be employed in standalone brake based safety systems such as ABS or ESP as well as integrated vehicle dynamics control systems [21].

\section{References}

1. Jiang, F., and Z. Gao. "An Application of Nonlinear PID Control to a Class of Truck ABS Problems." Decision and Control, 2001. Proceedings of the 40th IEEE Conference on. IEEE, 2001. 516-521.

2. Mauer, G. F. "A fuzzy logic controller for an ABS braking system." Fuzzy Systems, IEEE Transactions on 3, no. 4 (1995): 381-388.

3. Johansen, T. A., I. Petersen, J. Kalkkuhl, and J. Ludemann. "Gain-scheduled wheel slip control in automotive brake systems." Control Systems Technology, IEEE Transactions on 11, no. 6 (2003): 799-811.

4. Drakunov, S., U. Ozguner, P. Dix, and B. Ashrafi. "ABS control using optimum search via sliding modes." Control Systems Technology, IEEE Transactions on 3, no. 1 (1995): 79-85.

5. Savaresi, S. M., and M. Tanelli. Active braking control systems design for vehicles. Springer, 2010.

6. Youla, D., H. Jabr, and J. Bongiorno Jr. "Modern Wiener-Hopf design of optimal controllers--Part II: The multivariable case."
Automatic Control, IEEE Transactions on 21, no. 3 (1976): 319338.

7. Robert Bosch GmbH. Automotive Handbook. 8th. Edited by K. Reif. Cambridge, USA: Bentley Publishers, 2011.

8. Gunther Plapp. Electronic Brake Control Systems. Nov. 20, 2001.http://www.autospeed.com/cms/article.html?\&title=Electr onic-Brake-Control-Systems\&A=1202 (accessed April 22, 2014).

9. Van Zanten, A. T. "Evolution of electronic control systems for improving the vehicle dynamic behavior." In Proceedings of the 6th International Symposium on Advanced Vehicle Control. 2002. 1-9.

10. Schöner, H. P. "Automotive mechatronics." Control engineering practice 12, no. 11 (2004): 1343-1351.

11. Limpert, R. Brake Design and Safety. 3rd. USA: SAE International, 2011.

12. Kiencke, U., and L. Nielsen . Automotive Control Systems: For Engine, Driveline, and Vehicle, 2nd ed. Berlin: Springer-Verlag, 2005.

13. Van Zanten, A. T., R. Erhardt, G. Pfaff, F. Kost, U. Hartmann, and T. Ehret. "Control aspects of the Bosch-VDC." AVEC' 96. Achen, 1996.

14. Milliken, W. F., and D. L. Milliken. Race Car Vehicle Dynamics. USA: SAE International, 1995.

15. Assadian, F. "Neo-Classic Control Approach." Unpublished Lecture Notes. Cranfield University, 2011.

16. Assadian, F., "Mixed $\mathrm{H} \infty$ and Fuzzy Logic Controllers for the Automobile ABS," SAE Technical Paper 2001-01-0594, 2001, doi:10.4271/2001-01-0594.

17. Vidyasagar, M. Control system synthesis: a factorization approach. Morgan \& Claypool Publishers, 2011.

18. Doyle, J. C., B. A. Francis, and A. Tannenbaum. Feedback Control Theory. New York: Macmillan Publishing Co., 1992.

19. Skogestad, S., and I. Postlethwaite. Multivariable feedback control: analysis and design. 2. New York: Wiley, 2007.

20. Ogata, K. Modern Control Engineering. Prentice-Hall, 2010.

21. Soltani, A. M. Low Cost Integration of Enhanced Stability Control (ESC) with Electric Power Steering (EPS) (PhD Thesis). Cranfield University, 2014.

22. IPG Automotive GmbH. "CarMaker ${ }^{\circledR}$ Reference Manual Version 4.0.6." 2013.

\section{Contact Information}

Dr. Amir Soltani, Cranfield University, Cranfield, MK43 0AL, UK, $\underline{\text { m.m.soltani@Cranfield.ac.uk }}$

Prof. Francis Assaidan, University of California, Davis, USA, fassadian@udavis.edu

\section{Acknowledgments}

This work is undertaken within the Evoque_e project, co-funded by the UK's innovation agency, Innovate UK.

Page 8 of 8 
2015-04-14

New slip control system considering actuator dynamics

Soltani, Amirmasoud

SAE International

Soltani, A. and Assadian, F., (2015) New Slip Control System Considering Actuator Dynamics, SAE International Journal of Passenger Cars - Mechanical Systems, 8(2):512-520

http://dx.doi.org/10.4271/2015-01-0656

Downloaded from Cranfield Library Services E-Repository 